has the potential to be a safe, effective means of promoting psycho-social well-being in newly diagnosed patients and may also have positive effects for veteran patients. Further investigation into the use of one to one, peer support for brain tumour patients is an important research priority.

PS1 - 160

doi:10.1017/cjn.2016.353

\section{A Telephone Based Cognitive Assessment Tool for Brain Metastases Patients}

M.N. Tsao ${ }^{I}$, K. Edelstein, L.J. Bernstein, J. Wong, N. Laperriere, J.R. Perry, A. Sahgal, C. Menard, H. Soliman, E. Chow, T. Barnes, C. Danjoux, B.A. Millar, R. Wong, W. Wells, R.S. McIntyre, W. Mason, C. Chung

${ }^{1}$ University of Toronto, Toronto, ON may.tsao@sunnybrook.ca

Improving neurocognitive outcomes following treatment for brain metastases have become increasingly important. We propose that a brief telephone-based neurocognitive assessment may improve follow-up cognitive assessments in this palliative population. Aim: To prospectively assess the feasibility and reliability of a telephone based brief neurocognitive assessment compared to the same tests delivered face-to-face. Methods: Brain metastases patients to be treated with whole brain radiotherapy (WBRT) were assessed using a brief validated neurocognitive battery at baseline, at 1 month and 3 months following WBRT (in person and over the phone). The primary outcome was feasibility and inter-procedural (in person versus telephone) reliability. The secondary objective was to evaluate the change in neurocognitive function before and after WBRT. Results: Out of 39 patients enrolled, $82 \%$ of patients completed the baseline in-person and telephone neurocognitive assessments. However, at 1 month, only $41 \%$ of enrolled patients completed the in-person and telephone cognitive assessments and at 3 months, only $10 \%$ of patients completed them. Results pertaining to reliability and change in neurocognitive function will be updated. Conclusion: The pre-defined definition of feasibility (at least $80 \%$ completion for face to face and telephone neurocognitive assessments) was met at baseline. However, a large proportion of participants did not complete either telephone or in person neurocognitive follow-up at 1 month and at 3 months postWBRT. Attrition remained a challenge for neurocognitive testing in this population even when a telephone-based brief assessment was used.

PS1 - 163

doi:10.1017/cjn.2016.354

\section{Engaging in Difficult Discussions with Neuro-Oncology Patients: A Case Conference Based Quality Improvement Initiative}

C. Mueller ${ }^{\text {, }}$ D.B. Bilodeau, A.C. Chakraborty, J.E. Ellis, L.G. Gibson, E.I. Isenberg-Grzeda, C.M. Moroney, J.M. Myers, A.S. Scalco, A.S. Speke

${ }^{I}$ Sunnybrook Health Sciences Centre, Toronto, ON christina.mueller@sunnybrook.ca

Due to the non-curative nature of high grade brain tumours "difficult discussions" about goals of care, advance care planning, palliative care, and end of life are inherent to the practice of the neuro-oncology team. Clinician and patient barriers are common and may include anxiety about destroying hope, lack of readiness to discuss end of life topics, difficulty managing emotional responses, and concerns over competency in facilitating difficult discussions. Nonetheless, clinician comfort and skill in facilitating these discussions is critical and can impact patient perception of illness, coping response, and ability to make decisions about care. Methods At our regional cancer centre, a novel quality improvement initiative was designed to bring monthly case presentation of "difficult discussions" into a pre-existing weekly multi-disciplinary case conference (MCC). We will describe the rationale and developmental processes behind this initiative. Roughly 15 neuro-oncology clinicians attended each case conference, with guest attendance from palliative care and psychosocial oncology. Clinician groups represented included physicians $(77 \%)$, nurses $(8 \%)$, nurse practitioners $(10 \%)$, and occupational therapists (5\%). Baseline and monthly surveys were administered to determine clinicians' self- rated practices, skills, and attitudes towards "difficult discussions". Results Early findings indicate that the initiative has been well-received. Physicians indicated highest levels of agreement with the statement "I feel that having difficult discussions is part of my responsibility". Non physician groups indicated the greatest agreement that the intervention is beneficial to their practice. Discussion: Our challenges and successes may help guide others to incorporate a similar initiative at disease-site meetings.

PS1 - 188

doi:10.1017/cjn.2016.355

\section{Rehabilitation Consultation: An Integrated Model for Addressing Rehabilitation Concerns in the Primary Brain Tumor Population}

\author{
I. Lax ${ }^{1}$, M. Daniels, C. Kanter, W. Mason, K. Edelstein \\ ${ }^{I}$ University of Toronto, Toronto, $\mathrm{ON}$ \\ ilyse.lax@uhn.ca
}

Individuals with primary brain tumors experience a range of physical, cognitive and psychosocial sequelae which impact their independence, safety and quality of life. These impairments may be addressed through rehabilitation intervention. Despite acknowledgement that timely rehabilitation services over the course of the disease process is of benefit, few outpatient neurooncology treatment teams include a rehabilitation professional. Purpose: The aims are: (1) to describe a rehabilitation consultation model of care integrated into outpatient neuro-oncology treatment for individuals with primary brain tumors; and (2) to describe the characteristics of individuals referred for rehabilitation services. Methods: This retrospective descriptive study examined data from 200 individuals that received rehabilitation consultation from January 2015 to March 2016 at Princess Margaret Hospital, Pencer Brain Tumor Centre. Information on patient demographics, referral characteristics, and number of patient care visits was collected. Descriptive statistics were calculated. Preliminary Results: Of all patients, $(n=195)$, the most common diagnosis is glioblastoma, $39 \%(\mathrm{n}=76)$, and $50 \%$ are $50-69$ years of age $(\mathrm{M}=55, \mathrm{SD}=15.0)$. The most common reason for initial referral was decline in physical functioning, strength and balance (41\%). In $77 \%$ of cases, patients were seen immediately at the time of referral. In total, 540 consultations were completed (face-to-face $=230$, telephone $=$ 310 ) with 2.78 on average ( $\mathrm{SD}=4.0)$ per patient. Conclusion: Given the range of symptoms that individuals with primary brain 
tumors experience coupled with changes in functional status as the disease progresses, integrated and timely rehabilitation consultation is feasible.

\section{PEDIATRICS}

PS1 - 158

doi:10.1017/cjn.2016.356

\section{The Role of LIN28A in Neoplastic Transformation of Human Embryonic Stem Cells (hESCs)}

R. Kaur ${ }^{1}$, L. Liang, T. Werbowetski-Ogilvie

${ }^{I}$ University of Manitoba, Winnipeg, MA kaurr@myumanitoba.ca

Human embryonic stem cells (hESCs) are known for their indefinite self-renewal ability and pluripotent nature. However, during longterm culture, normal hESCs can undergo neoplastic transformation and acquire enhanced self-renewal ability and aberrant differentiation potential. These transformed-hESCs (trans-hESCs) exhibit high expression of the pluripotent gene, LIN28A. LIN28A, an RNA binding protein, is known: for its role in self-renewal of hESCs, as a reprogramming factor for generating inducedpluripotent stem cells and as a potent oncogene in several poorly differentiated, highly malignant human cancers. Despite its multiple functions, how LIN28A contributes to neoplastic transformation of normal hESCs is poorly understood. Our preliminary data demonstrate that following LIN28A knockdown, trans-hESCs display normal hESCs morphology consisting of both pluripotent colony cells surrounded by more differentiated fibroblast-like cells. Neural precursors derived from LIN28A knockdown trans-hESCs also revert back to a state of normal cell morphology and growth. Further analyses revealed that the expression levels of stage-specific embryonic antigen (SSEA3), OCT3/4 and NANOG decreases and are comparable to that observed in normal hESCs following LIN28A downregulation. Expression of miRNA targets of LIN28A such as let7i and mir125b was increased to levels seen in normal hESCs. These preliminary results indicate that LIN28A is a major contributing factor to neoplastic transformation of hESCs and that this process can be reversed by cellular "reprogramming". This study will enhance our understanding of role of LIN28A in the transformation process in various human cancers thus, underscoring the value of hESCs and their neoplastic-derivatives as cellular and molecular model for studying tumor progression.

PS1 - 169

doi:10.1017/cjn.2016.357

\section{Discovering the Treatment Refractory BTIC Population in Group 3 Medulloblastoma through Therapy Adapted Patient- Derived Human Mouse Xenograft (PDX) Model}

\section{Bakhshinyan ${ }^{I}$, T. Vijayakumar, B. Manoranjan, N. McFarlane, C. Venugopal, M. Singh, M. Qazi, P. Vora, S. Singh \\ ${ }^{1}$ McMaster University, Hamilton, ON bakhshd@mcmaster.ca}

Medulloblastoma (MB), the most common malignant pediatric brain tumor, is categorized into four molecular subgroups. Given the high rate of metastatic dissemination at diagnosis and recurrence in Group $3 \mathrm{MBs}$, these patients have the worst clinical outcome with a 5-year survivorship of approximately 50\%. By adapting the existing COG (Children's Oncology Group) Protocol for children with newly diagnosed high-risk $\mathrm{MB}$, for treatment of immuno-deficient mice intracranially engrafted with human $\mathrm{MB}$ brain tumour initiating cells we aim to identify and characterize the treatment-refractory cell population in Group $3 \mathrm{MBs}$. Mice were sacrificed at multiple time points during the course of tumor development and therapy: (i) at engraftment; (ii) post-radiation; (iii) post-radiation and chemotherapy; and (iv) at MB recurrence. MB cell populations recovered separately from brains and spines were comprehensively profiled for gene expression analysis, stem cell and molecular features to generate a global, comparative profile of MB cells through therapy. We report a higher expression of CD133, Sox2 and Bmi1 in addition to increased self-renewal capacity following chemoradiotherapy treatment. The enrichment map constructed from global gene expression analysis showed an increase in pathways regulating self-renewal, DNA repair and chemoresistance post-therapy despite the apparent decrease in tumour size and vascularity. Additionally, from gene expression at MB recurrence, we identified a list of genes that negatively correlate with survival in patients diagnosed with Group $3 \mathrm{MB}$. A differential genomic profile of the "treatment-responsive" tumors against those that fail therapy may contribute to discovery of novel therapeutic approaches for the most aggressive subgroup of MB.

PS1 - 170

doi:10.1017/cjn.2016.358

\section{Bmi1 is a Therapeutic Target in Recurrent Childhood Medulloblastoma}

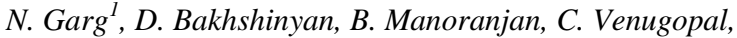 R. Hallett, S. Mahendram, T. Vijayakumar, M. Subapanditha, M. Qazi, M. Singh, N. McFarlane, A. Mann, P. Vora, T. Davis, S. Singh \\ ${ }^{I}$ McMaster University, Hamilton, ON ssingh@mcmaster.ca}

Medulloblastoma (MB) is the most common malignant pediatric brain tumour, and is categorized into four molecular subgroups, with Group $3 \mathrm{MB}$ having the worst prognosis due to the highest rate of metastatic dissemination and relapse. In this work, we describe the epigenetic regulator Bmil as a novel therapeutic target for treatment of recurrent Group $3 \mathrm{MB}$. Through comparative profiling of primary and recurrent $\mathrm{MB}$, we show that Bmil defines a treatment-refractory cell population that is uniquely targetable by a novel class of small molecule inhibitors. We have optimized an in vivo mouse-adapted therapy model that has the advantage of generating recurrent, human, treatment-refractory MBs. Our preliminary studies showed that although chemoradiotherapy administered to mice engrafted with human MB showed reduction in tumour size, Bmil expression was enriched in the post-treatment residual tumour. Furthermore, we found that knockdown of Bmi1 in human recurrent $\mathrm{MB}$ cells decreases proliferation and selfrenewing capacities of $\mathrm{MB}$ cells in vitro as well as both tumour size and extent of spinal leptomeningeal metastases in vivo. Oral administration of a potent Bmil inhibitor, PTC 028, resulted in a marked reduction in tumour burden and an increased survival in treatment cohort. Bmil inhibitors showed high specificity for MB cells and spared normal human neural stem cells, when treated with doses relevant for MB cells. As Group 3 medulloblastoma is often metastatic and uniformly fatal at recurrence, with no current or planned trials of targeted therapy, an efficacious agent such as Bmil inhibitor could be rapidly transitioned to clinical trials. 